\title{
Chapter 10 \\ Moiré Patterns Observed in Bi Layer Graphene Irradiated with High Energetic Protons
}

\author{
D. H. Galvan, A. Posada Amarillas, S. Mejía, C. Wing and M. José-Yacamán
}

\begin{abstract}
Customarily, it is likely that irradiated graphene yield indication of perturbations induced by irradiation. High Resolution Transmission Electron Microscopy (HRTEM) analysis has been performed on proton irradiated graphene. The analysis indicates the existence of Moiré patterns produced by the rotations induced by the irradiation in between planes. The rotations measured fluctuate between 3 and 5 degrees respectively. These rotations may influence the electronic properties of the material under investigation. In order to explain the observed rotations in between planes, theoretical analysis were performed under the scheme of extended Hückel tight-binding method. Average total energy of the system was careful analyzed throughout the experiment composed of two graphene layers with two carbon vacancies and then the replaced carbons were intercalated in between the two layers. The results obtained indicate that the system remain semi metallic. Moreover, the theoretical results yielded that the 3 degree rotation is favored, although the 5 degree rotation is not discarded. Furthermore, energy bands as well as total and projected DOS were performed in order to provide more information about the electronic changes induced by the rotations applied to the system.
\end{abstract}

D. H. Galvan ( $\square)$

Centro de Nanociencias y Nanotecnología-Universidad Nacional Autónoma de México, Apartado Postal 2681, CP 22800 Ensenada, BC, México

e-mail: donald@cnyn.unam.mx

P. O. 439036, 92143, San Ysidro, CA, USA

A. P. Amarillas

Departamento de Investigación en Física, Universidad de Sonora,

Apartado Postal 5-088, CP 83190 Hermosillo, SON, México

S. Mejía

Facultad de Ciencias Físico-Matemáticas, Universidad Autónoma de Nuevo León, CP 66451 San Nicolas de los Garza, NL, México

D. H. Galvan · M. José-Yacamán

Department of Chemical Engineering and International Center for Nanotechnology

\& Advanced Materials, University of Texas at Austin, Austin, TX 78712, USA

C. Wing

Instituto Nacional de Energía Nuclear, Ocoyoacac, Estado de México, México

J. M. Seminario (ed.), Design and Applications of Nanomaterials for Sensors,

Challenges and Advances in Computational Chemistry and Physics 16,

DOI 10.1007/978-94-017-8848-9_10, (C) Springer Science+Business Media Dordrecht 2014 


\subsection{Introduction}

The study of radiation damage was extensively studied in the past because the interest in fission reactors. Recently, a renewed interest in radiation damage studies has been driven by the need to expand new sources of energy and avoid environmental effects of hydrocarbons. Indeed, the development of advanced nuclear reactors is linked to the development of materials capable to operate under higher irradiation fluxes $[1,4,5,9,16,26]$.

Graphene is the name given to a monolayer of carbon atoms packed in a two dimensional honeycomb lattice. This system shows a peculiar effect, reminiscent of a Relativistic Quantum Hall effect [20], which trigger the scientific curiosity for the fundamental physics behind its behavior. Graphene showed unusual properties derived from its reduced dimensionality as well as its small size, which yielded an enormous potential to be used in ultra fast electronic transistors and switches.

Graphene shows a very high conductivity and an unusual Hall Effect.

In order to form a single hexagonal sheet of graphene, it could be built by means of two intercalated networks A and B, added to the interaction with the conduction electrons (electrons or holes) of each atom, create a new quasi-particle with special characteristics that they carry charge but without mass. These characteristics permit the quasi particle to travel inside the lattice, with a velocity of the order of 300 times less than the velocity of light and at the same times, without dispersion.

In this work we undertake the study of graphite in the form of HOPG (Highly Oriented Pyrolytic Graphite) subject to a highly energetic proton beam. It has been shown before $[3,7,10]$, that irradiation of HOPG using a high flux electron beam, produces breaking of the basal plane, disordering and even some tilt in the sample. In this case the main radiation damage is produced by the electron beam. In the present case, we used a proton beam of $5 \mathrm{MeV}$ and a total dose of $2.4 \times 10^{8} \mathrm{~Gy}$. The samples are then studied by Scanning Transmission Electron Microscopy (STEM) methods, which have minimum additional radiation. In particular we used bright field images in the STEM mode.

We found that as result of the irradiation, the graphene layers on the surface are rotated along an axis perpendicular to the graphite basal plane. Moreover, a rotation of about three degrees is often observed. We calculated energy bands and density of states of the rotated layer, and found some changes of the electronic properties of graphene.

\subsection{Experimental Methods}

Gold nanoparticles passivated with 1-dodecanethiol were synthesized by the Brust method reported elsewhere $[8,13]$. Three milligrams of the passivated gold particles were mixed with $100 \mathrm{mg}$ of graphite in $0.5 \mathrm{~mol}$ of toluene and then mounted over a brass sample holder. The solvent was allowed to evaporate until a dry pellet of the sample was formed. 
Samples were irradiated in an EN Tandem Van de Graaff accelerator, with a proton beam of $5 \mathrm{MeV}$. The beam was oriented along the basal plane of the sample, and total dose of $2.4 \times 10^{8} \mathrm{~Gy}$. was used. Afterwards, samples were cleaved using a scotch tape until an electron transparent film was obtained [19, 20]. Optical methods were used to verify the sample thickness. Then the sample was placed on a lacey carbon copper grid for transmission electron microscopy (TEM) analysis. The samples were analyzed using a JEOL $2010 \mathrm{~F}$ and Tecnai microscopes equipped with a Schottky-type field emission gun, ultra-high resolution pole piece $(\mathrm{Cs}=0.5 \mathrm{~mm})$, and an Scanning-Transmission (STEM) unit with a high angle annular dark field detector (HAADF) operating at $200 \mathrm{kV}$. A Gatan CCD camera was used for image acquisition. Images were obtained either, using an annular aperture in the STEM mode or in the HRTEM using the optimum focus condition. HRTEM simulated images were obtained using multi slice calculations.

\subsection{Theoretical Calculations}

The calculations were carried out by means of the tight-binding method [27] within the Extended Hückel [14] framework using YAeHMOP computer package [12, 18]. It is good to stress that the Extended Hückel method is a semi empirical approach for solving Schrödinger equation for a system of electrons, based on the variational theorem. In this approach, explicit electron correlation is not considered except for the intrinsic contributions included in the parameter set which were obtained from S. Alvarez et al. [2] or from the most accurate $a b$ initio calculations. The calculations were performed in the supercomputer Altix 350 using the input files accordingly to each specific case.

These calculations were performed on a system selected as a repeated cluster originated from a super cell. The super cell was generated from an infinite single sheet of carbon atoms, as depicted in Fig. 10.1a, which aroused from crystalline

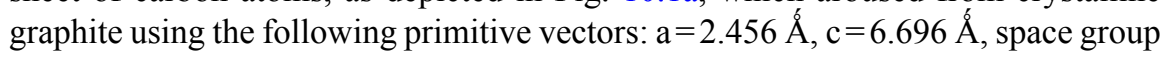
$186[15]$.

To prepare our theoretical sample, an infinite hexagonal honeycomb arrangement of carbon atoms were considered as depicted in Fig. 10.1a. To make the calculations simpler, a graphite single sheet made of 40 carbon atoms as depicted in Fig. $10.1 \mathrm{~b}$ were considered and in which 2 carbon atoms ( 3 and 13) were removed, as it would be a likely case of the effect of the proton irradiated beam on the sheet of carbon atoms. Moreover, these two carbon atoms were located at a distance of $1.67 \AA$ from the top layer and intercalated in between the second sheet, following a duplication of the second sheet and constructed of 40 carbon atoms in the AA configuration in order to create the $2 \mathrm{~L}$-graphene. The former configuration was made with the purpose of simulating a real scenario if $2 \mathrm{~L}$-graphene were subjected to proton irradiation. 
Fig. 10.1 a Band structure calculations for graphene 2-L, with two carbon vacancies intercalated in between layers. b Band structure calculations for graphene 2-L, with vacancies and intercalated. One of the layers (surface) has been rotated by three degrees

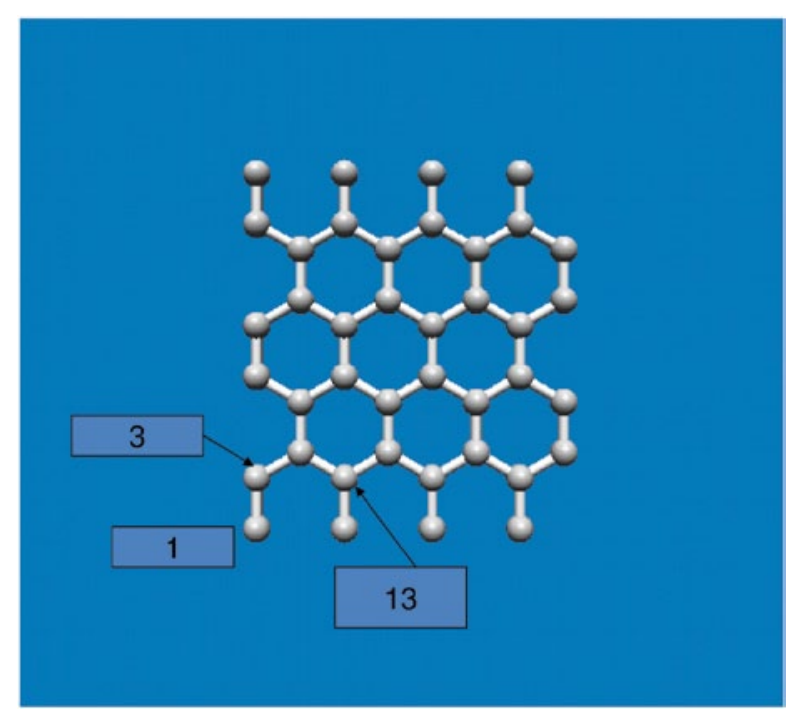

\subsection{Results and Discussion}

The samples of proton irradiated graphene were observed using TEM. A typical image is observed in Fig. 10.2a. A disordered structure can be observed. The structure can be revealed using micro diffraction. The nano diffraction mode of the microscope was used to obtain the patterns of different regions; a typical pattern is shown in Fig. 10.2b. As it is possible to see, the pattern is formed by two rotated hexagonal arrays of spots. The most common average angle was 3 degrees. In a few cases a larger rotation of $\sim 5$ degrees was observed.

Several groups [17, 21, 24, 25, 29] have reported that by using Scanning Tunneling Microscopy on carbon samples superlattices which they related to be Moiré patterns. On the other hand Rong et al. [24, 25] reported that by STM a hexagonal superlattice which show that it could be related to be a Moiré produced by the rotation of the top most plane with respect to the other layer. The rotation reported was 2.1. Furthermore, Xhie et al. [29] reported anomalous super lattices on (HOPG) using STM. They attributed that the periodic features reported may be caused to Moiré patterns due to rotations of one plane of the crystal with respect to the underlying graphite single crystal. The reported angle of rotation in between planes was of the order of 3.5.

Figure 10.1a shows a simulated image of the disordered layer. A very well defined Moiré pattern is observed. The micro diffraction image of two layers rotated by 3 degrees is shown in Fig. 10.1b. A very good agreement with the experiment is obtained. HAADF-STEM images to estimate the thickness of the disordered layers was used. Indeed the intensity of this type of images depends on the mass-thickness of the sample, and is mainly due to thermal Diffuse Scattering. The intensity has an approximate dependence of the atomic number $\sim Z^{1.7}$ [22]. Regions near the edge of the sample consisted in few layers of graphene was estimated. 

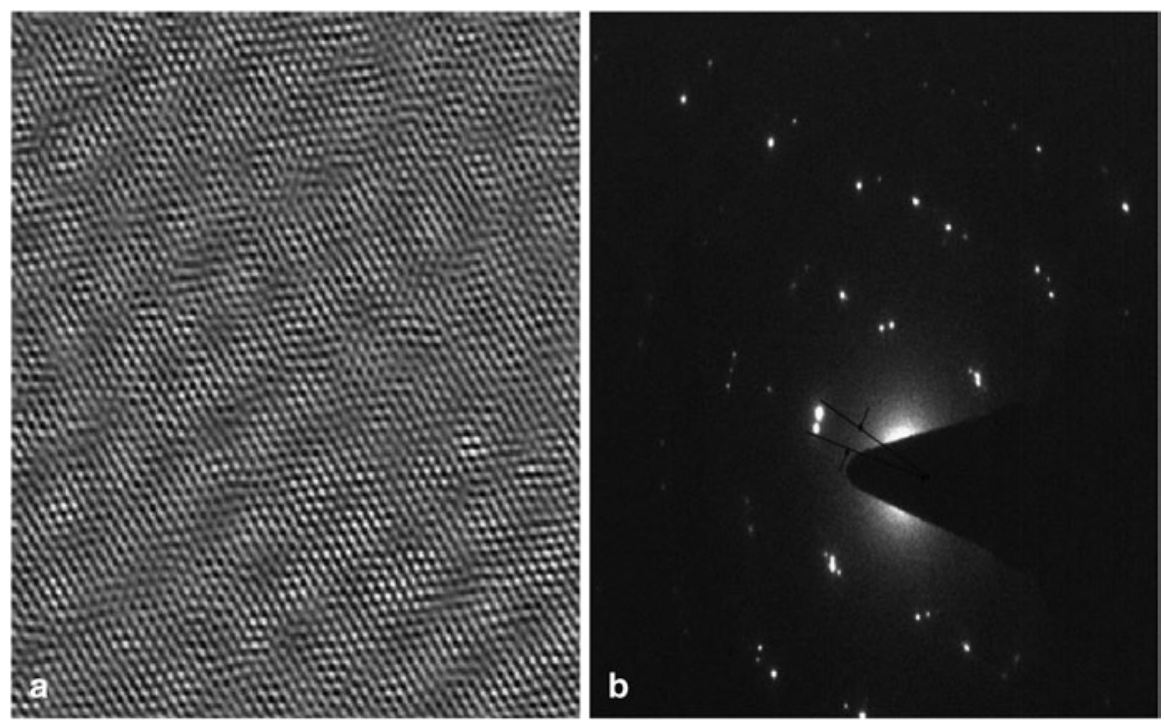

Fig. 10.2 a HRTEM micrograph for HOPG samples. b Diffraction patterns for the same material

In order to explain the results, we considered that the main damage produced by the protons is due to knock-on atom displacements [4]. A threshold energy of about 15-20 eV was estimated by Banhart et al. [4]. As a result of the high energy irradiation vacancies and interstitials will be formed. The mobility of the vacancies is low enough as to prevent rapid recombination.

In order to explain the diffraction patterns in 2-L rotated graphene with two carbon vacancies and intercalated in between layers (from now, this structure will be called as graphene 2-L), it proceed in the following way: Considering the average total energy of the system without rotation and after the rotation was applied, and taking into consideration that one of the layers is maintained fixed while the other layer is rotated by the appropriate amount around atom 1, see Fig. 10.3b. It is assumed that the interstitials are located between the layers and located $1.64 \AA$ from the top layer, rather than in the graphene plane.

Band structure calculations for 2-L unrotated graphene, 2-L graphene with applied rotatations by 3 and 5 , were calculated using $51 \mathrm{k}$-points for each case, and sampling the First Brillouin zone (FBZ) as depicted in Fig. 10.4a, b.

Atomic parameters for carbon atom used through the calculations were obtained from Alvarez et al. [2] and provided in Table 10.1. Experimental lattice parameters instead of optimized values were used searching for a best matching of our theoretical results with the available experimental information

The inset in Fig. 10.4 depicts the Wigner-Seitz cell for a hexagonal configuration used in the calculations. The Fermi level is indicated by a horizontal dotted line separating the valence band (VB) from the conduction band (CB) respectively. Energy in $\mathrm{eV} v s \mathrm{k}$-values (in the reciprocal space) were plotted for each case, ranging from $\Gamma\left(\begin{array}{lll}0 & 0 & 0\end{array}\right)$ to $\mathrm{M}(1 / 200)$ to $\mathrm{K}(1 / 31 / 30)$ of $(4 / \sqrt{ } 3)^{*}(\pi / \mathrm{a})$. 

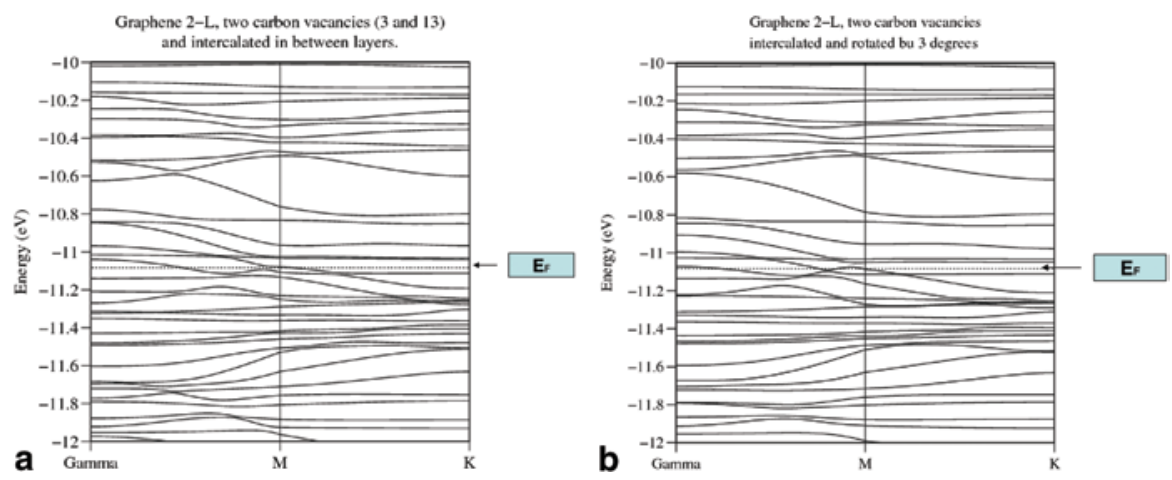

Fig. 10.3 A cell made out of 40 carbon atoms per layer was constructed

Fig. 10.4 Total and projected DOS for graphene 2-L, with vacancies and intercalated. The surface had been rotated by 3 degrees. Projected $\mathrm{C}$ 79-80 p-orbitals

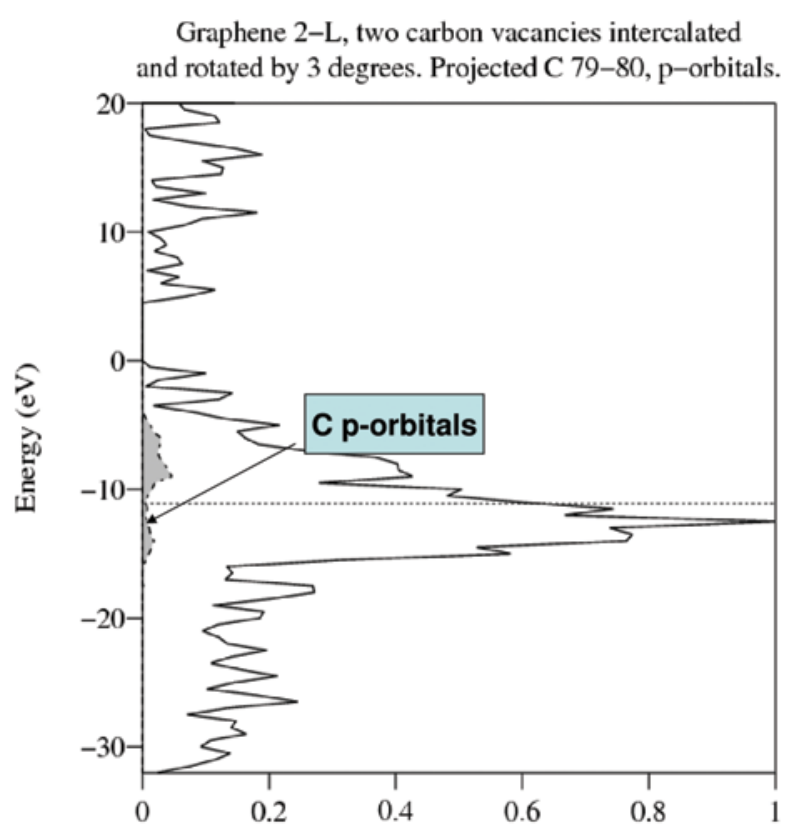

Table 10.2 provides information about the structure, Fermi energy location and average total energy $(\mathrm{eV})$. First, notice that for graphene 2-L unrotated, the average total energy is $-5600.6454 \mathrm{eV}$ and looking at Fig. 10.4a, the system has a semi metallic behavior due that two degenerate bands overlap at the $\mathrm{E}_{\mathrm{f}}$. Secondly, Fig. $10.4 \mathrm{~b}$ provides the energy bands when one of the layers (the one that we shall call surface which contains the two carbon vacancies) had been rotated by 3 degrees, the system behaves as a semi metal, also with three bands overlapping at the Fermi energy and with an average total energy of $-5600.9678 \mathrm{eV}$, similar to the unrotated case, which indicates that the rotation applied was not severe enough as to maintain the 
Table 10.1 Atomic parameters used in the extended Hückel tight-binding calculations, $\mathrm{H}_{\mathrm{ii}}(\mathrm{eV})$ and $\varsigma$ (Valence orbital ionization potential and exponent of Slater type orbitals). Each exponent is followed by a weighting coefficient in parentheses. A modified Wolfsberg-Helmholtz formula was used to calculate $\mathrm{H}_{\mathrm{ij}}$ [28]

\begin{tabular}{lllllll}
\hline Atom & Orbital & $\mathrm{H}_{\mathrm{ii}}$ & $\varsigma_{\mathrm{i} 1}$ & $\mathrm{C}_{1}$ & $\varsigma_{\mathrm{i} 2}$ & $\mathrm{C}_{2}$ \\
\hline $\mathrm{C}$ & $2 \mathrm{~s}$ & -21.4 & 1.62 & & & \\
& $2 \mathrm{p}$ & -11.40 & 1.62 & 0.0000 & 0.0000 & 0.0000 \\
\hline
\end{tabular}

Table 10.2 Structure, Fermi energy $(\mathrm{eV})$ and Total average energy $(\mathrm{eV})$ for the structures analyzed

\begin{tabular}{|c|c|c|c|}
\hline Structure & Fermi energy (eV) & Total average enegy $(\mathrm{eV})$ & Behavior \\
\hline $\begin{array}{c}\text { Graphene 2-L } \\
\text { nonrotated }\end{array}$ & $E_{f}=-11.2834$ & -5622.2462 & Semi metallic \\
\hline $\begin{array}{l}\text { Graphene } 2-\mathrm{L} \text { with } \\
\text { vacancies and } \\
\text { intercalated }\end{array}$ & $E_{f}=-11.0834$ & -5600.6454 & Semi metallic \\
\hline $\begin{array}{l}\text { Graphene } 2-\mathrm{L} \text { with } \\
\text { vacancies, interca- } \\
\text { lated and rotated } \\
\text { by } 3^{\circ}\end{array}$ & $E_{f}=-11.1004$ & -5600.9678 & Semi metallic \\
\hline $\begin{array}{l}\text { Graphene } 2-\mathrm{L} \text { with } \\
\text { vacancies, interca- } \\
\text { lated and rotated } \\
\text { by } 5^{\circ}\end{array}$ & $E_{f}=-11.1587$ & -5601.9677 & Semi metallic \\
\hline
\end{tabular}

system in a similar space group as the original one, favoring these rotated states. Furthermore, in the case when the applied rotation to the surface was 5 degrees, the system still is in the semi metallic state, but the total energy is of the order of $-5601.9677 \mathrm{eV}$, different to the unrotated and 3 degrees rotation applied. This indicates that the rotation applied was severe enough as to break the original space group producing states which are less likely favored.

Total and projected density of states (PDOS) for graphene 2-L with two carbon vacancies ( 3 and 13) intercalated, when $\mathrm{C} 1$ to 8 p-orbitals (for brevity) had been projected and depicted in Fig. 10.5a, it is necessary to point out that, it has been shown only the projection regarding to those atoms. Solid line represents total DOS (density of states), while the hatched dotted lines are the selected projected DOS for each orbital of the selected atoms. Horizontal dotted line indicates the Fermi level. Furthermore, concentration on those orbitals from the atoms which contributes most to the total DOS in the vicinity of $\mathrm{E}_{\mathrm{f}}$ was considered because they manifest their electronic behavior preferentially around that area. Notice that the main contribution to the total DOS comes from carbon $\pi$-contributions. It is necessary to stress that if all contributions from all the 78 carbon atoms were added, it will reproduce the total DOS (solid line). On the other hand Fig. 10.5b yield information regarding graphene $2-\mathrm{L}$ with two carbon vacancies ( 3 and 13) intercalated and then rotated (the surface layer) by $3^{0}$ rotation, when $\mathrm{C} 1$ to $8 \pi$-contributions (for brevity) had been projected notice small differences, when compared to the 
unrotated case, could be detected in the total DOS, but the overall contribution from the $\pi$-contributions are almost the same. These small differences could be attributed to the strain induced by rotation of one of the layers.

Moreover, Fig. 10.5c yielded information regarding carbon 79 and 80 (two carbon atoms displaced from the original location and intercalated in between the two layers). Notice that the $\pi$-contributions are negligible contributions to the total DOS at the Fermi level.

Due that the effect of rotation in between planes of graphene was considered, the effect is similar to the nanotubes subjected to distortion as reported by Rochefort et al. [23] they reported the effect of structural distortions of armchair carbon nanotubes on their electronic properties. One of the effects produced is to open of band gaps at some symmetric point of the FBZ, especially at the K point of the reciprocal space. The observed band gap was due to the asymmetric compression and dilation of the C-C bonds along NT. Moreover, they also reported that whenever they plot band gap $v s$ twisting angle a maximum band gap of $0.6 \mathrm{eV}$ occurred at 15 degrees

\subsection{Conclusions}

By comparing experimental results with quantum chemical calculations at the semi empirical level, it has been possible to explain that the disordered structures observed in proton irradiated graphene are due to rotation in the graphene honeycomb network. The calculations shown that the rotation of 3 degrees is favored producing a semi metallic behavior of the material in agreement to experimental results.

\section{References}

1. Ajayan PM, Ebbesen T (1997) Nanometric-size tubes of carbon. Rep Prog Phys 60(10):10251062

2. Alvarez S (March 1993) Tables of parameters for extended Hückel calculations. Universitat de Barcelona

3. Banhart F (1997) The transformation of graphitic onions to diamond under electron irradiation. J Appl Phys 81(8):3440-3445

4. Banhart F (1999) Irradiation effects in carbon nanostructures. Rep Prog Phys 62(8):1181-1221

5. Banhart F, Füller T, Redlich Ph, Ajayan PM (1997) The formation, annealing and self-compression of carbon onions under electron irradiation. Chem Phys Lett 269(3-4):349-355

6. Berger C, Song Z, Li T, Li X, Ogbazghi AY, Feng R, Dai Z, Marchenov AN, Conrad EH, First PN, de Heer WA (2004) Ultrathin epitaxial graphite: 2D electron gas properties and a route toward graphene-based nanoelectronics. J Phys Chem B 108(52):19912-19916

7. Berger C, Song Z, Li X, Wu X, Brown N, Naud C, Mayou D, Li T, Hass J, Marchenkov AN, Conrad EH, First PN, de Heer WA (2006) Electronic confinement and coherence in patterned epitaxial graphene. Science 312:1191-1196

8. Brust M, Walker M, Bethell D, Schiffrin DJ, Whyman R (1994) Synthesis of thiol-derivatised gold-nanoparticles, in a two-phase liquid-liquid system. J Chem Soc Chem Commun 801-802 
9. Burden PA, Hutchison JL (1996) Real-time observation of fullerene generation in a modified electron microscope. J Cryst Growth 158(1-2):185-188

10. Burden PA, Hutchison JL (1997) An investigation of the electron irradiation of graphite in a helium atmosphere using a modified electron microscope. Carbon 35(4):567-578

11. Geim AK, Novoselov KS (2007) The rise of graphene. Nat Mater 6:183-191

12. Glassey WV, Papoian GA, Hoffmann R (1999) The energy partition within one-electron formalism: a Hamiltonian population of study surface- $\mathrm{CO}$ interaction in the $\mathrm{c}(2 \times 2)-\mathrm{CO} / \mathrm{Ni}$ (100) chemisorption system. J Chem Phys 111(3)893-910

13. Gutierrez-Wing C, Santiago P, Ascencio JA, Camacho A, José-Yacamán M (2000) Self-assembling of gold nanoparticles in one, two and three dimensions. Appl Phys A, Mater Sci Process 71(3):237-243

14. Hoffmann R (1963) An extended Huckel theory. I. Hydrocarbons. J Chem Phys 39(6):13971412

15. Hoffmann U, Wilm DZ (1936) The crystal structure of graphite. Elektrochem 42:42

16. Koike J, Pedraza DF (1994) Dimensional changes in highly oriented pyrolytic graphite due to electron irradiation. J Mater Res 9(7):1899-1907

17. Kuwara M, Clarke DR, Smith DA (1990) Anomalous superperiodicity in scanning tunneling microscope images of graphite. Appl Phys Lett 56(24):2396-2398

18. Landrum GA The YAeHMOP package is freely available on www at: http://overlap.chem. Cornell.edu:8080/yaehmop.html. F orbitals are included in the calculations as version 3.0x, using W. V. Glassey's routine

19. Novoselov KS, Geim AK, Morozov SV, Jiang D, Zhang Y, Dubonos SV, Grigorieva IV, Firsov AA (2004) Electric field effect in atomically thin carbon films. Science 306:666-669

20. Novoselov KS, McCann E, Morozov SV, Fal'ko FI, Katsnelson MI, Zeitler U, Jiang D, Schedine F, Geim AK (2006) Unconventional quantum Hall effect and Berry's phase of $2_{\pi}$ in bilayered graphene. Nat Phys 2(3):177-180

21. Osing J, Shvets IV (1998) Bulk defects in graphite observed with a scanning tunneling microscope. Surf Sci 417(1):145-150

22. Peng Y, Nellist PD, Pennycook SJ (2004) HAADF-STEM imaging with sub-angstrom probes: a full Bloch wave analysis. J Electron Microsc 53(3):257-266

23. Rochefort A, Avouris P, Lesage F, Salahub DR (1991) Electrical and mechanical properties of distorted carbon nanotubes. Phys Rev B 60:13824-13830

24. Rong ZY (1994) Extended modifications of electronic structures caused by defects: scanning tunneling microscopy of graphite. Phys Rev B 50:1839-1843

25. Rong ZY, Kuiper P (1993) Electronic effects in scanning tunneling microscopy: Moiré pattern on a graphite surface. Phys Rev B 48:17427-17431

26. Thrower PA, Mayer RM (1978) Point defects and self-diffusion in graphene. Phys Status Solidi a 47(1):11-37

27. Whangbo MH, Hoffmann R (1978) The band structure of tetracyanoplatinate chain. J Am Chem Soc 100(19):6093-6098

28. Wolfsberg MW, Helmholtz L (1952) The spectra and electronic structure of the tetrahedral ions $\mathrm{MnO}_{4}^{-}, \mathrm{CrO}_{4}^{-}$, and $\mathrm{ClO}_{4}^{-}$. Chem Phys 20(5):837-843

29. Xhie J, Sattler K, Ge M, Venkateswaran N (1993) Phys Rev B 47:15835-15841 